\title{
X-RAY EMISSION FROM THE PLANETARY NEBULA NGC 1360
}

\author{
P A. J de Korte. J. J. Claas, F. A Jansen and \\ S P. McKechnie
}

Labonalor fol Space Research Leiden, Wassenaarsen'eg 78. PO Box 9504. 2300 RA Leiden. The Nerheslands

\begin{abstract}
The ExOSAT observatory has detected the nucleus of NGC 1360 in four photometric energy bands. The data rules out that the emisgion is from blackbody origin. Initial fits made with LTE model atmosphere spectra require the presence of highly ionized Oxygen and Neon in the stellar atmosphere.
\end{abstract}

\section{INTRODUCTION}

Central stars of planetary nebulae are believed to be a short stage in the evolution of a red glant towards a white dwarf. After ejection of the nebula the central star evolves

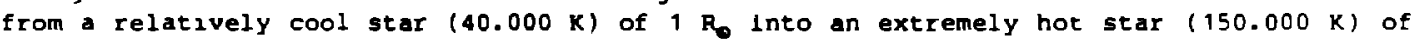
$0.01 \mathrm{R}$. Thereafter $2 t$ cools down to a white dwarf. Since the discovery of soft $x-r a y$ enigsion from the white dwarf $\mathrm{HZ} 43 / 1$ / numerous hot white dwarfs have been detected as $X$-ray sources. Because of their higher temperature, nucles of planetary nebulae are expected to be stronger $x$-ray sources, although sofar nothing has been published.

OBSERVATIONS

EXOSAT observations of NGC 1360 have been carried out on August 20, 1983. All five avallable filters have been used to obtain maximum spectral information and to be able to correct the data for UV-contamination. Source fluxes have been determined from a circular box of 2 arc minute diameter centered on the source. An annulus of 10 arc minute outer diameter around the source cell defines the background.

The data have been corrected for Uv-contamination by making use of inflight calibrations 12\%. For the determination of the contamination levels, the ANS-spectrum for NGC 1360 was used $/ 3 /$. Also an independent check has been made by using the internal consistency of all filter data. The UV-contamination for the data used in the $\mathrm{X}$-ray analysis is at most $7 \mathrm{q}$. Polypropylene data are, due to a higher uv-contamination, not used.

The corrected source fluxes together with $1 \mathrm{\sigma}$ errors are given in table I.

\section{ANALYSIS AND INTERPRETATION}

The $x$-ray flux is interpreted as surface emigsion from the central gtar of NGC 1360 . Another interpretation is that the emission originates from shocks induced by the stellar wind, which rams againgt the nebula. This can be excluded since the high resolution IUE-data don't show any aign of a stellar wind $/ 4 /$ and our data are consistent with emission from a point source.

The simplest approximation for emission from a stellar surface is blackbody radiation. Confidence levels in the $N_{h}$. $T_{b b}$ space have been determined through application of the 11 kelyhood ratio /5/. The 908 confidence contour is given in figure 1 . The source

Table I X-ray countrates for NGC 1360

\begin{tabular}{llll}
\hline Experiment & Filter & $\begin{array}{l}\text { Time } \\
\text { (sec) }\end{array}$ & $\begin{array}{l}\text { X-ray Elux } \\
\text { (counts/sec) }\end{array}$ \\
\hline LE1 & Al/Parylene & $\mathbf{5 7 0 6 . 4}$ & $0.008 \pm 0.0015$ \\
LE1 & Boron & $\mathbf{4 8 8 2 . 5}$ & $0.0054 \pm 0.0015$ \\
LE2 & 3000A Lexan & 3172.7 & $0.1834 \pm 0.0075$ \\
LE2 & 4000A Lexan & 4133.0 & $0.0834 \pm 0.0046$ \\
LE2 & Polypropylene & 3742.7 & $0.1660 \pm 0.0063$ \\
\hline
\end{tabular}




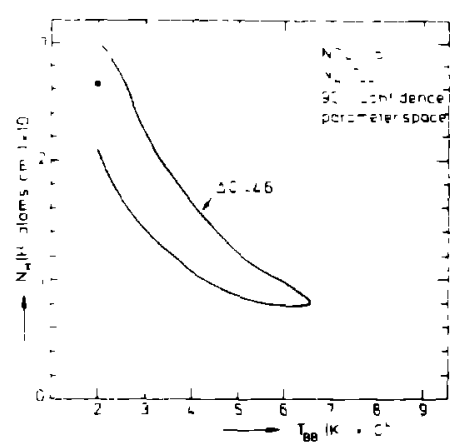

Figure 1: 90 confidence contour for a blackbody fit to the NGC 1360 x-ray data.

intensity, represented by $(R / D)^{2}$, is given for the $N_{h}$. $T_{b b}$ values on the 908 confidence contour in figure 2 .

For the interstellar column density in the direction of NGC1360, (1.4 \pm 0.1$): 020$ $\mathrm{H}-\mathrm{atcom} \theta / \mathrm{cm}^{2} / 6 /$, the blackbody temperature equals $38.000 \pm 8.000 \mathrm{~K}$. In cage $\mathrm{N}_{\mathrm{h}} 2 \mathrm{~s}$ left free the maximum allowed temperature is $65.000 \mathrm{~K}$. This value $1 \mathrm{~s}$ totally inconsistent with temperatures derived sofar. The He II Zanstra temperature equals $85.000 \mathrm{~K} / 7 /$, while colour temperatures derived from the optical and $U V$ continuum are between 100.000 and $120.000 \mathrm{~K}$ $13,4 /$. The only temperature close to ours is an effective temperature derived from non-LTE model atmosphere fits to absorption line profiles/8/, which results in an effective temperature equal to $65.000 \pm 15.000 \mathrm{~K}$.

The range of $(R / D)^{2}$ in figure 2 is compared with values derived on the basis of thy /7/ and also on the basis of the surface gravity, $\log g=5.2 / 8 /$, the distance $D=350 \mathrm{pc} / 7 /$. and a commonly accepted value for the stellar mass, $1 . e .0 .8 \mathrm{M}$. The (R/D) ${ }^{2}$ derived from the $x$-ray data 18 one or two orders of magnitude too high.

A blackbody origin can therefore clearly be ruled out. The X-ray data require a stellar flux consistent with a blackbody temperature of at least $85,000 \mathrm{~K}$, while its spectral shape should be as steep as for $38.000 \mathrm{~K}$.

In addition we have investigated a grid of LTE-atmospheres calculated by Humer and Mihalas 19/. These models include besides $H$ and $H e$ also $C, N, O$ and Ne which have absorption edges $1 n$ the $\mathrm{x}$-ray range.

In figure 3 model atmosphere spectra in the $x-r a y$ range are draw for an effective temperature of $100.000 \mathrm{~K}, \log \mathrm{g}=5$ and for three different elemental abundances, $2 . e$. the 200 (I), 300 (II) and 400 (III) model spectra series of Humer and Mihalas. The best fit 15 obtained for the highest abundance, l.e. the 400-series (Abundance III) spectrum, as indicated by the reduced chi-square. From the spectra in figure 3 it is clear that the

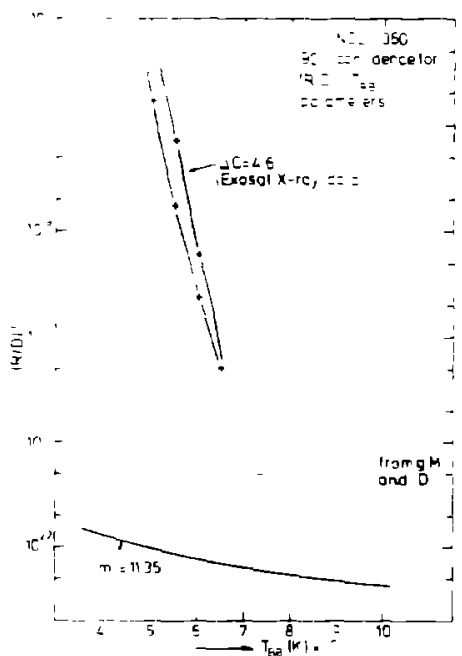

Figure 2: Luminosity, $(R, D)^{2}$, as a function of blackbody temperature for 908 confidence contour values of temperature and interstellar column density. 


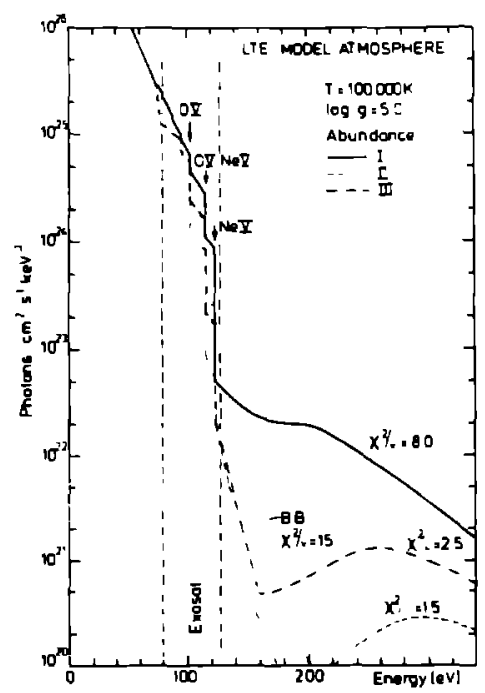

Figure 3: LTE model atmosphere spectra of Humer and Mihalas /9/ for $T_{\text {eff }}=100.000 \mathrm{~K}, \log g=5$ and three different abundances. The quality of the fit to the $\mathrm{X}$-ray data is given by a reduced chl-square.

required steepness of the spectrum in the range observed by Exosat is caused by the opacity of the $O \mathrm{~V}$ and $\mathrm{Ne} \mathrm{V}$ lons. Those opacities allow for a combination of a large $X$-ray flux, 1.e. high effective temperature, and a steep spectral shape in the spectral range observed.

For objects close to the Eddington 12mit, like NGC 1360, non-LTE model atmosphere spectra should however be used. Recently a grid of models have been published by Husfeld et al. 110\%. These calculations make only use of Hydrogen and Helium. Their spectral shape shortward of 228 A is even flatter than for a blackbody spectrum, so that they don't fit the X-ray data at all. Non-LTE model spectra including abundances of $C, N, O$ and Ne will be required to carry out a more quantative analysis on the available spectral data.

\section{DISCUSSION AND CONCLUSION}

The X-ray data cannot be represented by a blackbody spectrum. LTE model atmosphere spectra lead to good fits if opacitieg from $O V$ and Ne $V$ lons are included. The derivation of abundances for planetary nebula nuclei 25 very important. White dwarfs are known to have almost pure Hydrogen or Helium atmospheres,which 28 explained by gravitational settling during the cooling phase. Sofar abundances for planetary nebulae nuclei, just one evolution stage before that of the white dwarf, are unknown.

The $X$-ray measurements demonstrate the possibility to determine the oxygen and Neon abundance. Non LTE-calculations with temperature, Helium abundance and gravicy fixed to values obtained from optical line profiles $/ \theta /$ will be carried out in order to extract the abundances of Oxygen and Neon, from the avallable X-ray data.

\section{REFERENCES}

1. B. Margon, R. Malına, S. Bowyer, R. Cruddace, and M. Lampton: Ap.J., 203, L25 (1976)

2. P.A.J. de Korte, EXOSAT internal conmunication (1983)

3. S.R. Pottasch, P.R. Wesselius, C.C. Wu, H. Fleten, and R.J. van Duinen: Agtron. Astrophys. 62, 95 (1978)

4. R.P. Kudritzki, private communication (1984)

5. W.Cash; Ap.J. 228, 939 (1979)

6. C.Heiles: Astron. Astrophys. Suppl. 20, 37 (1975)

7. J.B. Kaler: Ap.J. 271 , 188 (1983)

8. R.H. Mendez, R.P. Kudrztzk1, J. Gruschinske, and K.P. Simon: Agtron. Astrophys. 101, $323(1981)$

9. D.G. Hummer, and D. Mrhalas: Mon. Not. R. agtr. Soc. 147, $339(1970)$

10. D. Husfeld, R.P. Kudritzk1, K.P. Simon, and R.E.S. Clegg: Astron. Astrophys. 134, 139 (1984) 